\title{
A Partitioning Methodology That Optimises the Area on Reconfigurable Real-Time Embedded Systems
}

\author{
Camel Tanougast \\ Laboratoire d'Instrumentation Electronique de Nancy, Université de Nancy I, BP 239, 54600 Vandoeuvre Lès Nancy, France
}

Email: tanougast@lien.u-nancy.fr

Yves Berviller

Laboratoire d'Instrumentation Electronique de Nancy, Université de Nancy I, BP 239, 54600 Vandoeuvre Lès Nancy, France Email: berville@lien.u-nancy.fr

\author{
Serge Weber \\ Laboratoire d'Instrumentation Electronique de Nancy, Université de Nancy I, BP 239, 54600 Vandoeuvre Lès Nancy, France \\ Email: sweber@lien.u-nancy.fr
}

\author{
Philippe Brunet \\ Laboratoire d'Instrumentation Electronique de Nancy, Université de Nancy I, BP 239, 54600 Vandoeuvre Lès Nancy, France \\ Email:brunet@lien.u-nancy.fr
}

Received 27 February 2002 and in revised form 12 September 2002

\begin{abstract}
We provide a methodology used for the temporal partitioning of the data-path part of an algorithm for a reconfigurable embedded system. Temporal partitioning of applications for reconfigurable computing systems is a very active research field and some methods and tools have already been proposed. But all these methodologies target the domain of existing reconfigurable accelerators or reconfigurable processors. In this case, the number of cells in the reconfigurable array is an implementation constraint and the goal of an optimised partitioning is to minimise the processing time and/or the memory bandwidth requirement. Here, we present a strategy for partitioning and optimising designs. The originality of our method is that we use the dynamic reconfiguration in order to minimise the number of cells needed to implement the data path of an application under a time constraint. This approach can be useful for the design of an embedded system. Our approach is illustrated by a reconfigurable implementation of a real-time image processing data path.
\end{abstract}

Keywords and phrases: partitioning, FPGA, implementation, reconfigurable systems on chip.

\section{INTRODUCTION}

The dynamically reconfigurable computing consists in the successive execution of a sequence of algorithms on the same device. The objective is to swap different algorithms on the same hardware structure, by reconfiguring the FPGA array in hardware several times in a constrained time and with a defined partitioning and scheduling [1,2]. Several architectures have been designed and have validated the dynamically reconfigurable computing concept for the real-time processing $[3,4,5]$. However, the mechanisms of algorithms optimal decomposition (partitioning) for runtime reconfiguration (RTR) is an aspect in which many things remain to do. Indeed, if we analyse the works in this domain, we can see that they are restricted to the application development approach
[6]. We observe that: firstly, these methods do not lead to the minimal spatial resources. Secondly, a judicious temporal partitioning can avoid an oversizing of the resources needed [7].

We discuss here the partitioning problem for the RTR. In the task of implementing an algorithm on reconfigurable hardware, we can distinguish two approaches (Figure 1). The most common is what we call the application development approach and the other is what we call the system design approach. In the first case, we have to fit an algorithm, with an optional time constraint, in an existing system made of a host CPU connected to a reconfigurable logic array. In this case, the goal of an optimal implementation is to minimise one or more of the following criteria: processing time, memory bandwidth, number of reconfigurations. In the second case, 


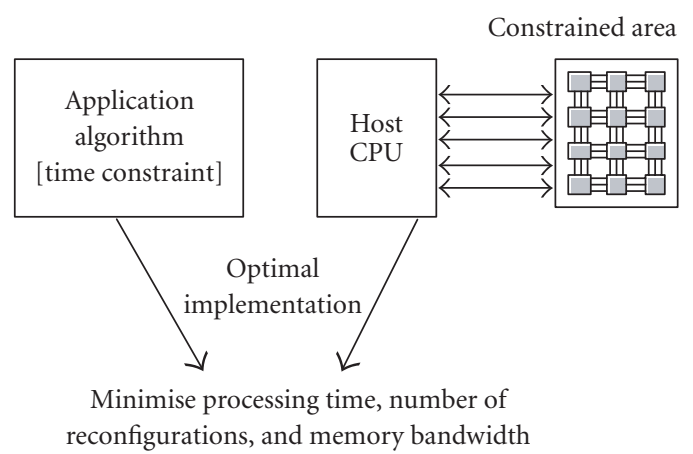

(a) Application development.

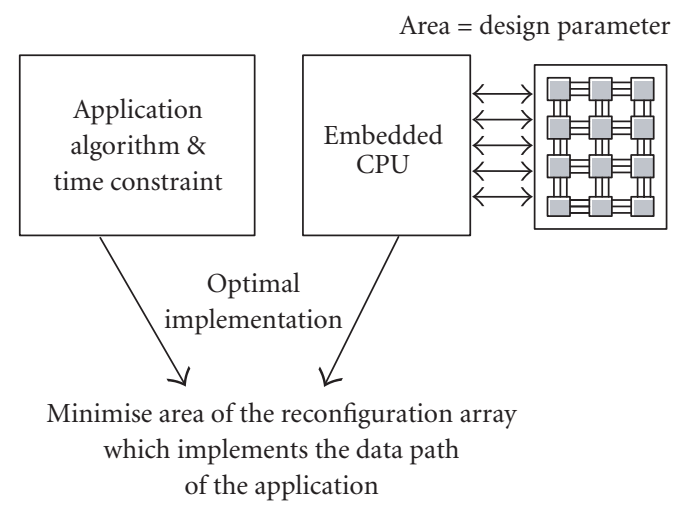

(b) Application-specific design.

FIGURE 1: The two approaches used to implement an algorithm on reconfigurable hardware.

however, we have to implement an algorithm with a required time constraint on a system which is still under the design exploration phase. The design parameter is the size of the logic array which is used to implement the data-path part of the algorithm. Here, an optimal implementation is the one that leads to the minimal area of the reconfigurable array.

Embedded systems can take several advantages of the use of FPGAs. The most obvious is the possibility to frequently update the digital hardware functions. But we can also use the dynamic resources allocation feature in order to instantiate each operator only for the strict required time. This permits to enhance the silicon efficiency by reducing the reconfigurable array's area [8]. Our goal is the definition of a methodology which allows to use RTR, in the architectural design flow, in order to minimise the FPGA resources needed for the implementation of a time-constrained algorithm. So, the challenge is double. Firstly to find trade-offs between flexibility and algorithm implementation efficiency through the programmable logic array coupled with a host CPU (processor, DSP, etc.). Secondly to obtain a computeraided design techniques for optimal synthesis which include the dynamic reconfiguration in an implementation.

Previous advanced works exist in the field of temporal partitioning and synthesis for RTR architectures $[9,10,11$, $12,13,14]$. All these approaches assume the existence of a resources constraint. Among them, there is the GARP project [9]. The goal of GARP is the hardware acceleration of loops in a $C$ program by the use of the data-path synthesis tool GAMA [10] and the GARP reconfigurable processor. The SPARCS project $[11,12]$ is a CAD tool suite tailored for application development on multi-FPGAs reconfigurable computing architectures. The main cost function used here is the data memory bandwidth. In [13], one also proposes both a model and a methodology to take the advantages of common operators in successive partitions. A simple model for specifying, visualizing, and developing designs, which contains elements that can be reconfigured in runtime, has been proposed. This judicious approach allows to reduce the configuration time and the application execution time. But we need additional logic resources (area) to realize an implementation with this approach. Furthermore, this model does not include the timing aspects in order to satisfy the real-time and it does not specify the partitioning of the implementation.

These interesting works do not pursue the same goal as we do. Indeed, we try to find the minimal area which allows to meet the time constraint and not the minimal memory bandwidth or execution time which allows to meet the resources constraint. We address the system design approach. We search the smallest sized reconfigurable logic array that satisfies the application specification. In our case, the intermediate results between each partition are stored in a draft memory (not shown in Figure 1).

An overview of the paper is as follows. In Section 2, we provide a formal definition of our partitioning problem. In Section 3, we present the partitioning strategy. In Section 4, we illustrate the application of our method with an image processing algorithm. In this example, we apply our method in an automatic way while showing the possibility of evolution which could be associated. In Sections 5 and 6, we discuss the approach, conclude, and present future works.

\section{PROBLEM FORMULATION}

The partitioning of the runtime reconfiguration real-time application could be classified as a spatiotemporal problem. Indeed, we have to split the algorithm in time (the different partitions) and to define spatially each partition. It is a time-constrained problem with a dynamic resource allocation in contrast with the scheduling of runtime reconfiguration [15]. Then, we make the following assumptions about the application. Firstly, the algorithm can be modelled as an acyclic data-flow graph (DFG) denoted here by $G(V, E)$, where the set of vertices $V=\left\{O_{1}, O_{2}, \ldots, O_{m}\right\}$ corresponds to the arithmetic and logical operators and the set of directed edges $E=\left\{e_{1}, e_{2}, \ldots, e_{p}\right\}$ represents the data dependencies between operations. Secondly, The application has a critical time constraint $T$. The problem to solve is the following.

For a given FPGA family, we have to find the set $\left\{P_{1}, P_{2}, \ldots, P_{n}\right\}$ of subgraphs of $G$ such that

$$
\bigcup_{i=1}^{n} P_{i}=G
$$


and which allows to execute the algorithm by meeting the time constraint $T$ and the data dependencies modelled by $E$ and requires the minimal amount of FPGA cells. The number of FPGA cells used, which is an approximation of the area of the array, is given by (2), where $P_{i}$ is one among the $n$ partitions,

$$
S=\max _{i \in\{1, \ldots, n\}}\left(\operatorname{Area}\left(P_{i}\right)\right) .
$$

The FPGA resources needed by a partition $i$ is given by (3), where $M_{i}$ is the number of elementary operators in partition $P_{i}$ and $\operatorname{Area}\left(O_{k}\right)$ is the amount of resources needed by operator $\mathrm{O}_{k}$,

$$
\text { Area }\left(P_{i}\right)=\sum_{k \in\left\{1, \ldots, M_{i}\right\}} \operatorname{Area}\left(O_{k}\right)
$$

The exclusion of cyclic DFG application is motivated by the following reasons.

(i) We assume that a codesign prepartitioning step allows to separate the purely data path part (for the reconfigurable logic array) from the cyclic control part (for the CPU). In this case, only the data path will be processed by our RTR partitioning method.

(ii) In the case of small feedback loops (such as for IIR filters), the partitioning must keep the entire loop in the same partition.

\section{TEMPORAL PARTITIONING}

The general outline of the method is shown in Figure 2. It is structured in three parts. In the first, we compute an approximation of the number of partitions (blocks A, B, C, D in Figure 2), then we deduce their boundaries (block E), and finally we refine, when it is possible, the final partitioning (blocks E, F).

\subsection{Number of partitions}

In order to reduce the search domain, we first estimate the minimum number of partitions that we can achieve and the quantity of resources allowed in a partition. To do this, we use an operator library which is target dependent. This library allows to associate two attributes to each vertex of the graph $G$. These attributes are $t_{i}$ and $\operatorname{Area}\left(O_{i}\right)$, respectively, the maximal path delay and the number of elementary FPGA cells are needed for operator $O_{i}$. These two quantities are functions of the size (number of bits) of the data to process. If we know the size of the initial data to process, it is easy to deduce the size at each node by a "software execution" of the graph with the maximal value for the input data.

Furthermore, we make the following assumptions.

(i) The data to process are grouped in blocks of $N$ data.

(ii) The number of operations to apply to each data in a block is deterministic (i.e., not data dependant).

(iii) We use pipeline registers between all nodes of the graph.

(iv) We consider that the reconfiguration time is given by rt(target), a function of the FPGA technology used. (v) We neglect the resources needed by the read and write counters (pointers) and the small-associated state machine (controller part). In our applications, this corresponds to a static part. The implementation result will take into account this part in the summary of needed resources (see Section 4). by

Thus, the minimal operating time period to $\mathrm{max}_{\max }$ is given

$$
\text { to }_{\max }=\max _{i \in\{1, \ldots, m\}}\left(t_{i}\right)
$$

and the total number $C$ of cells used by the application is given by

$$
C=\sum_{i \in\{1, \ldots, m\}} \operatorname{Area}\left(O_{i}\right)
$$

where $\{1, \ldots, m\}$ is the set of all operators of data path $G$. Hence, we obtain the minimum number of partitions $n$ as given by (6) and the corresponding optimal size $C_{n}$ (number of cells) of each partition by (7),

$$
\begin{gathered}
n=\frac{T}{(N+\sigma) \cdot \mathrm{to}_{\max }+\mathrm{rt}()}, \\
C_{n}=\frac{C}{n},
\end{gathered}
$$

where $T$ is the time constraint (in seconds), $N$ the number of data words in a block, $\sigma$ the total number of latency cycles (prologue + epilogue) of the whole data path, to $\mathrm{max}_{\max }$ the propagation delay of the slowest operator in the DFG in seconds and it corresponds to the maximum time between two successive vertices of graph $G$ thanks to the full pipelined process, and $\mathrm{rt}($ ) the reconfiguration time. In the case of the partially reconfigurable FPGA technology, rt() can be approximated by a linear function of the area of the functional units being downloaded. The expression of $\mathrm{rt}()$ is the following:

$$
\operatorname{rt}()=\frac{C}{V}
$$

where $V$ is the configuration speed (cells/s) of the FPGA, and $C$ the number of cells required to implement the entire DFG. We consider that each reconfiguration overwrites the previous partition (we configure a number of cells equal to the size of the biggest partition). This guarantees that the previous configuration will never interfere with the current configuration. In the case of the fully reconfigurable FPGA technology, the $\mathrm{rt}($ ) function is a constant depending on the size of FPGA. In this case, $\mathrm{rt}()$ is a discrete linear function increasing in steps, corresponding to the different sized FPGAs. The numerator of (6) is the total allowed processing time (time constraint). The left side expression of the denominator is the effective processing time of one data block (containing $N$ data) and the right-side expression is the time loosed to load the $n$ configurations (total reconfiguration time of $G$ ).

In most application domains like image processing (see Section 4), we can neglect the impact of the pipeline latency time in comparison with the processing time $(N \gg \sigma)$. So, in the case of partially reconfigurable FPGA technology, we 


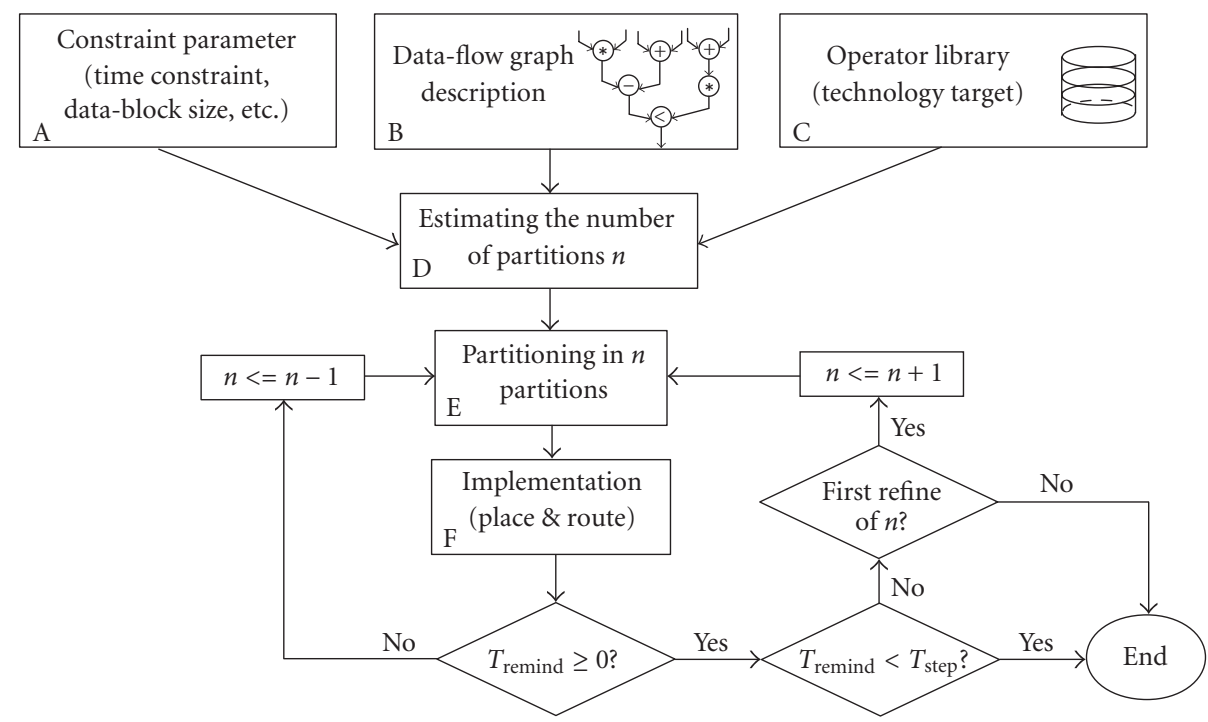

FIGURE 2: General outline of the partitioning method.

can approximate (6) by (9) (corresponding to the block D in Figure 2),

$$
n \approx \frac{T}{N \cdot \text { to }_{\max }+C / V} .
$$

The value of $n$ given by (9) is a pessimistic one (worst case) because we consider that the slowest operator is present in each partition.

\subsection{Initial partitioning}

A pseudoalgorithm of the partitioning scheme is given as,

$$
\begin{aligned}
& G<=\text { data-flow graph of the application } \\
& P_{1}, P_{2}, \ldots, P_{n}<=\text { empty partitions } \\
& \text { for } i \text { in }\{1, \ldots, n\} \\
& \qquad \begin{array}{l}
C<=0 \\
\text { while } C<C_{n} \\
\text { append }\left(P_{i} \text {, First_Leave }(G)\right) \\
\qquad<=C+\text { First_Leave }(G) \cdot \text { Area } \\
\text { remove }(G \text {, First_Leave }(G))
\end{array}
\end{aligned}
$$$$
\text { end while }
$$

end for

We consider a First_Leave() function that takes a DFG as an argument and which returns a terminal node. We cover the graph from the leaves to the root(s) by accumulating the sizes of the covered nodes until the sum is as close as possible to $C_{n}$. These covered vertices make the first partition. We remove the corresponding nodes from the graph and we iterate the covering until the remaining graph is empty. The partitioning is then finished.

There is a great degree of freedom in the implementation of the First_Leave() function, because there are usually many leaves in a DFG. The unique strong constraint is that the choice must be made in order to guarantee the data dependencies across the whole partition. The reading of the leaves of the DFG can be random or ordered. In our case, it is ordered. We consider $G$ as a two-dimensional table containing parameters related to the operators of the DFG. The First_Leave() is carried out in the reading order of the table, containing the operator arguments of the DFG (left to right). The first aim of the First_Leave() function is to create partitions with area as homogeneous as possible. At this time, the First_Leave() does not care about memory bandwidth.

\subsection{Refinement after implementation}

After the placement and routing of each partition that was obtained in the initial phase, we are able to compute the exact processing time. It is also possible to take into account the value of the synthesized frequency close to the maximal processing frequency for each partition.

The analysis of the gap between the total processing time (configuration and execution) and the time constraint permits to make a decision about the partitioning. If it is necessary to reduce the number of partitions or possible to increase it, we return to the step described in Section 3.2 with a new value for $n$. Else the partitioning is considered as an optimal one (see Figure 2).

\section{APPLICATION TO IMAGE PROCESSING}

\subsection{Algorithm}

We illustrate our method with an image processing algorithm. This application area is a good choice for our approach because the data is naturally organized in blocks (the images), there are many low-level processing algorithms which can be modelled by a DFG, and the time constraint is usually the image acquisition period. We assume that the 


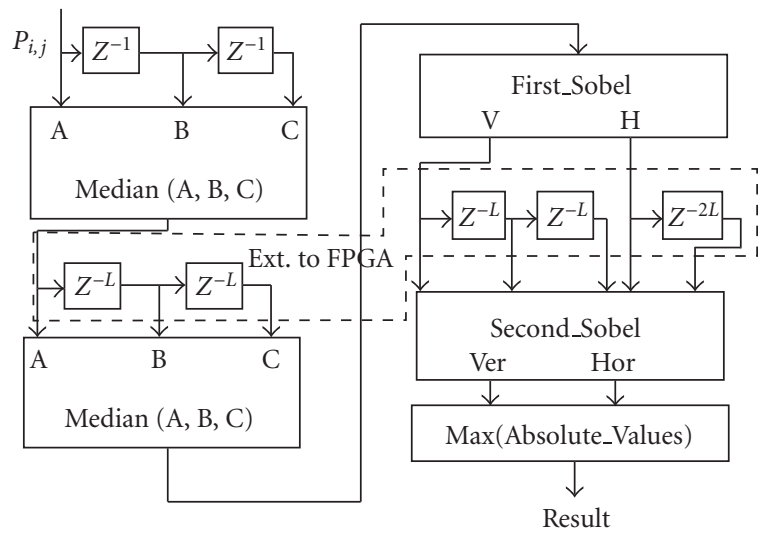

Figure 3: General view of images edge detector.

images are taken at a rate of 25 per second with a spatial resolution of $512^{2}$ pixels and each pixel grey level is an eight bits value. Thus, we have a time constraint of 40 milliseconds.

The algorithm used here is a $3 \times 3$ median filter followed by an edge detector and its general view is given in Figure 3. In this example, we consider a separable median filter [16] and a Sobel operator. The median filter provides the median value of three vertical successive horizontal median values. Each horizontal median value is simply the median value of three successive pixels in a line. This filter allows to eliminate the impulsion noise while preserving the edges quality. The principle of the implementation is to sort the pixels in the $3 \times 3$ neighborhood by their grey level value and then to use only the median value (the one in the 5 th position on 9 values). This operator is constituted of eight bits comparators and multiplexers. The gradient computation is achieved by a Sobel operator. This corresponds to a convolution of the image by successive application of two monodimensional filters. These filters are the vertical and horizontal Sobel operator, respectively. The final gradient value of the central pixel is the maximum absolute value from vertical and horizontal gradient. The line delays are made with components external to the FPGA (Figure 3).

\subsection{DFG annotation}

The FPGA family used in this example is the Atmel AT40K series. These FPGAs have a configuration speed of about 1365 cells per millisecond and have a partial reconfiguration mode. The analysis of the data sheet [17] allows us to obtain the characteristics given in Table 1 for some operator types. In this table, $T_{\text {cell }}$ is the propagation delay of one cell, $T_{\text {rout }}$ is the intraoperator routing delay, and $T_{\text {setup }}$ is the flip-flop setup time. From the characteristics given in the data sheet [17], we obtain the following values as a first estimation for the execution time of usual elementary operators (Table 2).

In practice, there is a linear relationship between the estimated execution time and the real execution time which integrate the routing time needed between two successive nodes. This is shown in Figure 4 which is a plot of the estimated execution time versus the real execution time for some different
TABLE 1: Usual operator characterization (AT40K).

\begin{tabular}{|c|c|c|}
\hline $\begin{array}{l}D \text {-bit } \\
\text { operator }\end{array}$ & $\begin{array}{c}\text { Number of } \\
\text { cells }\end{array}$ & Estimated execution time \\
\hline $\begin{array}{l}\text { Multiplication or } \\
\text { division by } 2^{k}\end{array}$ & 0 & 0 \\
\hline $\begin{array}{l}\text { Adder or } \\
\text { subtractor }\end{array}$ & $D+1$ & $D \cdot\left(T_{\text {cell }}+T_{\text {rout }}\right)+T_{\text {setup }}$ \\
\hline Multiplexer & $D$ & $T_{\text {cell }}+T_{\text {setup }}$ \\
\hline Comparator & $2 \cdot D$ & $(2 \cdot D-1) \cdot\left(T_{\text {cell }}\right)+2 \cdot T_{\text {rout }}+T_{\text {setup }}$ \\
\hline $\begin{array}{l}\text { Absolute value } \\
\text { (two's complement) }\end{array}$ & $D-1$ & $D \cdot\left(T_{\text {cell }}+T_{\text {rout }}\right)+T_{\text {setup }}$ \\
\hline $\begin{array}{l}\text { Additional } \\
\text { synchronization } \\
\text { register }\end{array}$ & $D$ & $T_{\text {cell }}+T_{\text {setup }}$ \\
\hline
\end{tabular}

TABLE 2: Estimated execution time of some eight-bit operators in AT40K technology.

\begin{tabular}{lc}
\hline Eight-bit operators & Estimated execution time (ns) \\
\hline Comparator & 27.34 \\
\hline Multiplexer & 5 \\
\hline Absolute value & 22.07 \\
\hline Adder, subtractor & 16.46 \\
\hline $\begin{array}{l}\text { Combinatory logic with } \\
\text { interpropagation logic cell }\end{array}$ & 17 \\
\hline $\begin{array}{l}\text { Combinatory logic without } \\
\text { interpropagation logic cell }\end{array}$ & 5 \\
\hline
\end{tabular}

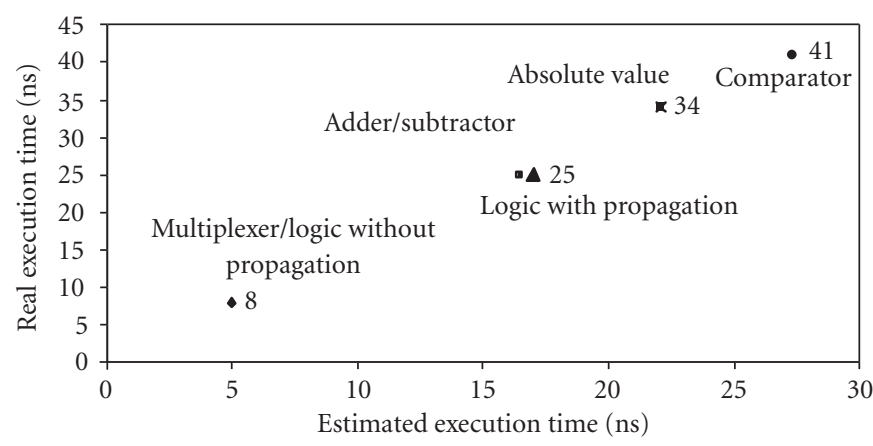

FIGURE 4: Estimated time versus real execution time of some operators in AT40K technology.

usual low-level operators. Those operators have been implemented individually in the FPGA array between registers. This linearity remains true when the operators are wellaligned in a strict cascade. This relationship is not valid for specialised capabilities already hardwired in the FPGAs (such as RAM block, multiplier, etc.). From this observation, we can obtain an approximation of the execution times of the operators contained in the data path. The results are more 

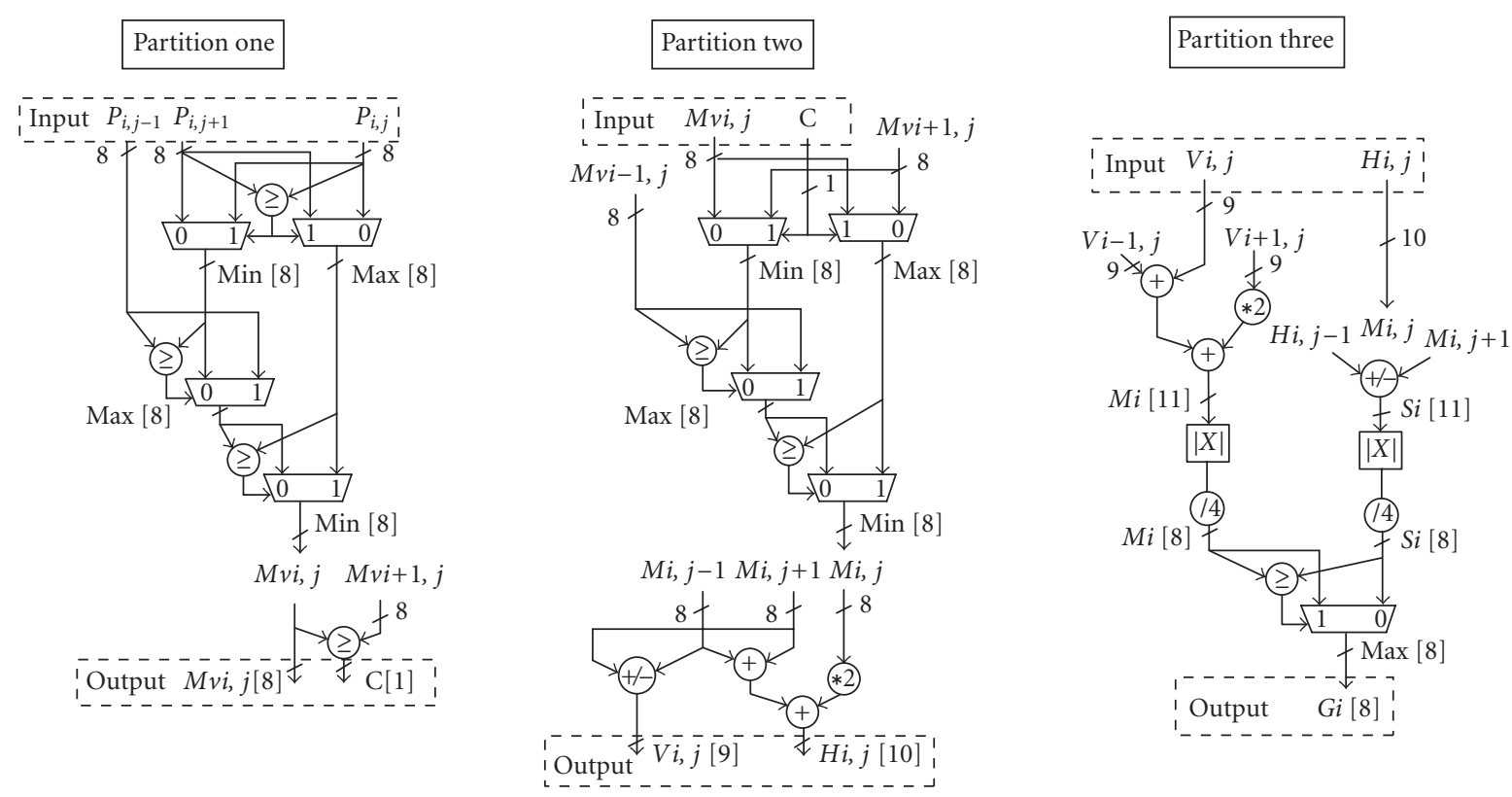

FIgURe 5: Partitioning used to implement the image edge detector DFG.

exact as the algorithm is regular such as the data path (strict cascade of the operators).

The evaluation of the routing in the general case is difficult to realize. The execution time after implementation of a regular graph does not depend on the type of operator. A weighting coefficient binds the real execution time with the estimated one. This coefficient estimates the routing delay between operators based on the estimated execution time. With these estimations and by taking into account the increase of data size caused by processing, we can annotate the DFG. Then, we can deduce the number and the characteristics of all the operators. For instance, in Table 3 we give the data about the algorithm example. In this table, the execution time is an estimation of the real execution time. From the data, we deduce the number of partitions needed to implement a dedicated data path in an optimised way. Thus, for the edges detector, among all operators of the data path, we can see that the slowest operator is an eight-bit comparator and that we have to reconfigure 467 cells. Hence, from (9) (result of block D), we obtain a value of three for $n$. The size of each partition $\left(C_{n}\right)$ that implement the global data path should be about 156 cells. Table 4 summarizes the estimation for an RTR implementation of the algorithm. By applying the method described in Section 3, we obtain a first partitioning represented in Figure 5 (result of block E).

\subsection{Implementation results}

In order to illustrate our method, we tested this partitioning methodology on the ARDOISE architecture [5]. This platform is constituted of AT40K FPGA and two $1 \mathrm{MB}$ SRAM memory banks used as draft memory. Our method is not aimed to target such architectures with resources constraint. Nevertheless, the results obtained in terms of used resources
TABle 3: Number and characteristics of the operators of the edge detector (on AT40K).

\begin{tabular}{lcccc}
\hline Operators & Quantity & $\begin{array}{c}\text { Size } \\
\text { (bits) }\end{array}$ & $\begin{array}{c}\text { Area } \\
\text { (cells) }\end{array}$ & $\begin{array}{c}\text { Execution } \\
\text { time (ns) }\end{array}$ \\
\hline Comparator & 7 & 8 & 16 & 41 \\
\hline Multiplexer & 9 & 8 & 8 & 8 \\
\hline Absolute value & 2 & 11 & 10 & 34 \\
\hline Subtractor & 1 & 8 & 9 & 25 \\
\hline & 1 & 10 & 11 & 30.5 \\
Adder & 1 & 8 & 9 & 25 \\
& 2 & 9 & 10 & 27.5 \\
Multiplication & 1 & 10 & 11 & 30.5 \\
by 2 & 2 & 8 & 0 & routing \\
\hline Division by 4 & 2 & 9 & 0 & routing \\
\hline $\begin{array}{l}\text { Register } \\
\text { (pipeline or }\end{array}$ & 13 & 8 & 8 & routing \\
delay) & 4 & 9 & 9 & 8 \\
& 5 & 10 & 10 & \\
\hline
\end{tabular}

TABLE 4: Resources estimation for the image edge detector.

\begin{tabular}{lcccc}
\hline $\begin{array}{l}\text { Total } \\
\text { area } \\
(\text { cells })\end{array}$ & $\begin{array}{l}\text { Operator } \\
\text { execution } \\
\text { time }(\mathrm{ns})\end{array}$ & $\begin{array}{c}\text { Step } \\
\text { Number } \\
(n)\end{array}$ & $\begin{array}{c}\text { Area by } \\
\text { step } \\
(\text { cells })\end{array}$ & $\begin{array}{l}\text { Reconfiguration } \\
\text { time by step }(\mu \mathrm{s})\end{array}$ \\
\hline 467 & 41 & 3 & 156 & 114 \\
\hline
\end{tabular}

and working frequency are still valid for any AT40K-like array. The required features are a small logic cell granularity, 
TABLE 5: Implementation results in an AT40K of edges detector.

\begin{tabular}{lcccc}
\hline $\begin{array}{l}\text { Partition } \\
\text { number }\end{array}$ & $\begin{array}{l}\text { Number } \\
\text { of cells }\end{array}$ & $\begin{array}{c}\text { Operator } \\
\text { execution } \\
\text { time }(\mathrm{ns})\end{array}$ & $\begin{array}{c}\text { Partition } \\
\text { reconfiguration } \\
\text { time }(\mu \mathrm{s})\end{array}$ & $\begin{array}{c}\text { Partition } \\
\text { processing } \\
\text { time }(\mathrm{ms})\end{array}$ \\
\hline 1 & 152 & 40.1 & 111 & 10.5 \\
\hline 2 & 156 & 40.3 & 114 & 10.6 \\
\hline 3 & 159 & 36.7 & 116 & 9.6 \\
\hline
\end{tabular}

one flip-flop in each cell, and the partial configuration possibility. Table 5 summarizes the implementation results of edges detector algorithm (result of block F). We notice that a dynamic execution in three steps can be achieved in real time. This is in accordance with our estimation (Table 4).

We can note that a fourth partition is not feasible (second iteration of blocks $\mathrm{E}$ and $\mathrm{F}$ is not possible, see Figure 2), because the allowed maximal operator execution time would be less than 34 nanoseconds. Indeed, if we analyse the time remaining, we find that one supplementary partition does not allow to realise the real-time processing. The maximal number of cells by partition allows to determine the functional density gain factor obtained by the runtime reconfiguration implementation [8]. In this example, the gain factor in terms of functional density is approximately three in contrast with the global implementation of this data path (static implementation) for real-time processing. This gain is obtained without accounting for the controller part (static part). Figure 5 represents each partition successively implemented in the reconfigurable array for the edges detector.

There are many ways to partition the algorithm with our strategy. Obviously, the best solution is to find the partitioning that leads to the same number of cells used in each step. However, in practice, it is necessary to take into account the memory bandwidth bottleneck. That is why the best practical partitioning needs to keep the data throughput in accordance with the performances of the used memory.

Generally, if we have enough memory bandwidth, we can estimate the cost of the control part in the following way. The memory resources must be able to store two images (we assume a constant flow processing), memory size of $256 \mathrm{~KB}$. The controller needs two counters to address the memories, a state machine for the control of the RTR and the management of the memories for read or write access. In our case, the controller consists in two 18-bit counters $\left(N=512^{2}\right.$ pixels), a state machine with five states, a 4-bit register to capture the number of partitions (we assume a number of reconfiguration lower than 16), a counter indicating the number of partitions, a 4-bit comparator, and a not-operator to indicate which alternate buffer memory we have to read and write. With the targeted FPGA structure, the logic area of the controller in each configuration stage requires a number of resources of 49 logical cells. If we add the controller area to the resource needed for our example, we obtain a computing area of 209 cells with a memory bandwidth of 19 bits.

\section{DISCUSSION}

We can compare our method to the more classical architectural synthesis, which is based on the reuse of operator by adding control. Indeed, the goal of the two approaches is the minimization of hardware resources. When architectural synthesis is applied, the operators must be dimensioned for the largest data size even if such a size is rarely processed (generally only after many processing passes). Similarly, even if an operator is not frequently used, it must be present (and thus consumes resources) for the whole processing duration. These drawbacks, which do no more exist for a runtime-reconfigurable architecture, generate an increase in logical resources needs. Furthermore, the resources reuse can lead to increased routing delay if compared to a fully spatial data path, and thus decrease the global architecture efficiency. But, if we use the dynamic resources allocation features of FPGAs, we instantiate only the needed operators at each instant (temporal locality [6]) and assure that the relative placement of operators is optimal for the current processing (functional locality [6]).

Nevertheless, this approach has also some costs. Firstly, if we consider the silicon area, an FPGA needs between five and ten times more silicon than a full custom ASIC (ideal target for architectural synthesis) at the same equivalent gates count and with lower speed. But this cost is not too important if we consider the ability to make big modifications of the hardware functions without any change of the hardware part. Secondly, in terms of memory throughput, with respect to a fully static implementation, our approach requires an increase of a factor of at least the number of partitions $n$. Thirdly, in terms of power consumption, both approaches are equivalent if we neglect both the over clocking needed to compensate for reconfiguration durations and consumptions outside the FPGA. Indeed, in a first approximation, power consumption scales linearly with processing frequency and functional area (number of toggling nodes), and we multiply the first by $n$ and divide the second by $n$. But, if we take into account the consumption due to memory $\mathrm{read} /$ writes and the reconfigurations themselves, then our approach performs clearly less good.

\section{CONCLUSION AND FUTURE WORK}

We propose a method for the temporal partitioning of a DFG that permits to minimise the array size of an FPGA by using the dynamic reconfiguration feature. This approach increases the silicon efficiency by processing at the maximally allowed frequency on the smallest area and which satisfies the realtime constraint. The method is based, among other steps, on an estimation of the number of possible partitions by use of a characterized (speed and area) library of operators for the target FPGA. We illustrate the method by applying it on an images processing algorithm and by real implementation on the ARDOISE architecture.

Currently, we work on more accurate resources estimation which takes into account the memory management part of the data path and also checks if the available memory 
bandwidth is sufficient. We also try to adapt the First_ Leave() function to include the memory bandwidth. Our next goal is to adjust the first estimation of partitioning in order to keep the compromise between homogeneous areas and memory bandwidth minimization. At this time, we have not automated the partition search procedure, which is roughly a graph covering function. We plan to develop an automated tool like in GAMA or SPARCS. We also study the possibilities to include an automatic architectural solutions exploration for the implementation of arithmetic operators.

\section{REFERENCES}

[1] S. A. Guccione and D. Levi, "Design advantages of runtime reconfiguration," in Reconfigurable Technology: FPGAs for Computing and Applications, J. Schewel, P. M. Athanas, S. A. Guccione, S. Ludwig, and J. T. McHenry, Eds., vol. 3844 of SPIE Proceedings, pp. 87-92, SPIE, Bellingham, Wash, USA, September 1999.

[2] P. Lysaght and J. Dunlop, "Dynamic reconfiguration of FPGAs," in More FPGAs, W. Moore and W. Luk, Eds., pp. 82-94, Abingdon EE\&CS Books, Oxford, England, 1994.

[3] M. J. Wirthlin and B. L. Hutchings, "A dynamic instruction set computer," in Proc. IEEE Workshop on FPGAs for Custom Computing Machines, pp. 99-107, Napa, Calif, USA, April 1995.

[4] S. C. Goldstein, H. Schmit, M. Budiu, S. Cadambi, M. Moe, and R. Taylor, "PipeRench: A reconfigurable architecture and compiler," IEEE Computer, vol. 33, no. 4, pp. 70-77, 2000.

[5] D. Demigny, M. Paindavoine, and S. Weber, "Architecture reconfigurable dynamiquement pour le traitement temps réel des images," TSI, vol. 18, no. 10, pp. 1087-1112, 1999.

[6] X. Zhang and K. W. Ng, "A review of high-level synthesis for dynamically reconfigurable FPGAs," Microprocessors and Microsystems, vol. 24, no. 2000, pp. 199-211, 2000.

[7] C. Tanougast, Méthodologie de partitionnement applicable aux systèmes sur puce à base de FPGA, pour l'implantation en reconfiguration dynamique d'algorithmes flot de données, Ph.D. thesis, Université de Nancy I, Vandoeuvre, France, 2001.

[8] M. J. Wirthlin and B. L. Hutchings, "Improving functional density using run-time circuit reconfiguration," IEEE Transactions on Very Large Scale Integration (VLSI) Systems, vol. 6, no. 2, pp. 247-256, 1998.

[9] T. J. Callahan, J. Hauser, and J. Wawrzynek, "The GARP architecture and C compiler," IEEE Computer, vol. 33, no. 4, pp. 62-69, 2000.

[10] T. J. Callahan, P. Chong, A. DeHon, and J. Wawrzynek, "Fast module mapping and placement for data paths in FPGAs," in Proc. ACM/SIGDA International Symposium on Field Programmable Gate Arrays, pp. 123-132, Monterey, Calif, USA, February 1998.

[11] I. Ouaiss, S. Govindarajan, V. Srinivasan, M. Kaul, and R. Vemuri, "An integrated partitioning and synthesis system for dynamically reconfigurable multi-FPGA architectures," in Parallel and Distributed Processing, vol. 1388 of Lecture Notes in Computer Science, pp. 31-36, Springer-Verlag, Orlando, Fla, USA, 1998.

[12] M. Kaul and R. Vemuri, "Optimal temporal partitioning and synthesis for reconfigurable architectures," in Int. Symposium on Field-Programmable Custom Computing Machines, pp. 312-313, Napa, Calif, USA, April 1998.

[13] W. Luk, N. Shirazi, and P. Y. K. Cheung, "Modelling and optimizing run-time reconfiguration systems," in IEEE Symposium on FPGAs for Custom Computing Machines, K. L. Pocek and J. Arnold, Eds., pp. 167-176, IEEE Computer Society Press, Napa Valley, Calif, USA, April 1996.

[14] M. Karthikeya, P. Gajjala, and B. Dinesh, "Temporal partitioning and scheduling data flow graphs for reconfigurable computers," IEEE Trans. on Computers, vol. 48, no. 6, pp. 579$590,1999$.

[15] M. Vasilko and D. Ait-Boudaoud, "Scheduling for dynamically reconfigurable FPGAs," in Proc. International Workshop on Logic and Architecture Synthesis, IFIP TC10 WG10.5, pp. 328-336, Grenoble, France, December 1995.

[16] N. Demassieux, Architecture VLSI pour le traitement d'images: Une contribution à l'étude du traitement matériel de l'information, Ph.D. thesis, École Nationale Supérieure des Télécommunications (ENST), Paris, France, 1991.

[17] Atmel AT40k datasheet, Rev. 0896A-A-12/97.

Camel Tanougast received his Ph.D. degree in microelectronic and electronic instrumentation from the University of Nancy I, France, in 2001. Currently, he is a researcher in Electronic Instrumentation Laboratory of Nancy (LIEN). His research interests include design and implementation of real-time processing architecture, FPGA design, and the terrestrial digital television (DVB-T).

Yves Berviller received the Ph.D. degree in electronic engineering in 1998 from the Henri Poincaré University, Nancy, France. $\mathrm{He}$ is currently an Assistant Professor at Henri Poincaré University. His research interests include computing vision, system on chip development and research, FPGA design, and the terrestrial digital television (DVB-T).

Serge Weber received the Ph.D. degree in electronic engineering, in 1986, from the University of Nancy (France). In 1988, he joined the Electronics Laboratory of Nancy (LIEN) as an Associate Professor. Since September 1997, he is Professor and Manager of the Electronic Architecture group at LIEN. His research interests include reconfigurable and parallel architectures for image and signal processing or for intelligent sensors.

Philippe Brunet received his M.S. degree from the University of Dijon, France in 2001. Currently, he is a Ph.D. research student in electronic engineering at the Electronic Instrumentation Laboratory of Nancy (LIEN), University of Nancy 1 . His main interest concerns design FPGA and computing vision.
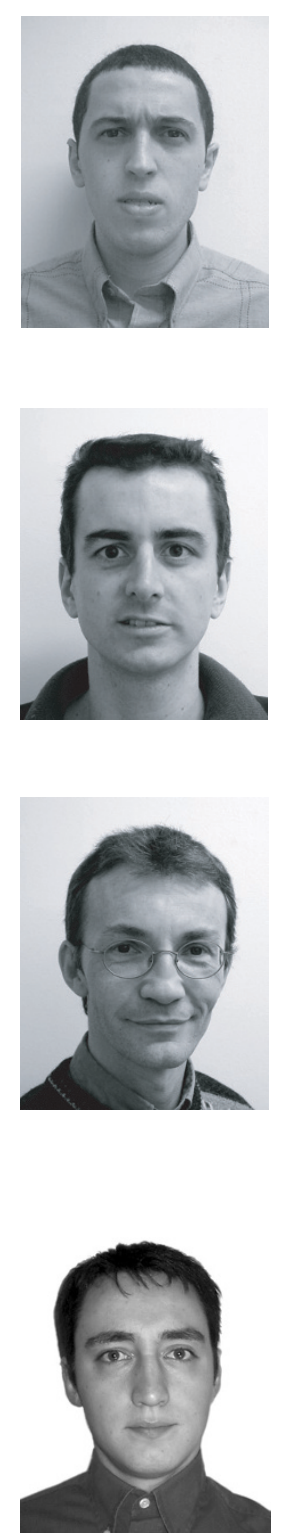